\title{
A new class of polymeric monodentate phosphoramidite ligands for asymmetric hydrogenation of $\alpha$-dehydroamino acid derivatives
}

\author{
Meng Yang, Ji Zhang, Na Wang, and Xiao-Qi Yu* \\ Key Laboratory of Green Chemistry and Technology (Ministry of Education), Department of \\ Chemistry, Sichuan University, Chengdu, 610064, P. R. China \\ E-mail:xqyu@tfol.com
}

\begin{abstract}
A new class of rigid and sterically regular polymeric monodentate phosphoramidite ligands derived from $(R)$-BINOL were prepared and applied in the Rhodium-catalyzed asymmetric hydrogenation of $\alpha$-dehydroamino acid derivatives. High catalytic activities and enantioselectivities (up to $99 \%$ ee) were achieved, which are comparable to those obtained with MonoPhos. The optimal mol ratio of $\mathrm{Rh} /$ ligand was proved to be $1: 1$.
\end{abstract}

Keywords: Asymmetric hydrogenation, monophosphine ligands, polymer, binol

\section{Introduction}

Transition-metal-catalyzed asymmetric hydrogenation of prochiral olefins is an important method for achieving highly enantiopure molecules, which were widely used in pharmacy and fine chemicals industries. ${ }^{1,2}$ Chiral phosphine ligands, especially bisphosphorus derivatives, have been widely used for asymmetric hydrogenation. ${ }^{3}$ More recently, due to the readily accessibility, high stability and excellent performance in the catalytic reactions, ${ }^{4}$ many chiral monophosphine ligands such as phosphonites, phosphites and phosphoramidites have been emploied in asymmetric hydrogenation of functionalized olefins showing high enantioselectivities. ${ }^{5}$ However, for most homogeneous catalysis systems, the separation of the catalyst was a problem. Some polymer-supported chiral monophosphine catalysts have been developed for asymmetric hydrogenation to overcome the difficulties. ${ }^{6}$ In the classical approaches, these polymer-supported ligands were usually obtained by tethering phosphine ligands with a flexible and linear polymer backbone such as polyethylene glycol or polystyrene. The mutual winding or self-twisting caused by the flexibility of polymer backbone might lead to the change of the microenvironment of the active centers. Compared to the corresponding homogeneous ligands, polymer-supported catalysts often displayed reduced enantioselectivity and less efficiency in the catalysis. ${ }^{7,8}$ As an improvement, dendrimer supported phosphoramidite ligands have been developed and successfully applied in asymmetric hydrogenations. ${ }^{9}$ 
$\mathrm{Pu}{ }^{10-13}$ has developed a novel main-chain chiral conjugated polymers derived from $(R)$ BINOL and studied their applications in asymmetric catalysis reactions. This new type of polymers possessed a high degree of structural rigidity, therefore the steric and electronic environments of BINOL were difficult to be altered. In this paper, we report the synthesis of a new class of polymeric monodentate phosphoramidites ligands derived from $(R)$-BINOL and their applications in the rhodium-catalyzed asymmetric hydrogenation of $\alpha$-dehydroamino acid derivatives.

\section{Results and Discussion}

\section{Preparation of ligands}

The preparation of polymeric monodentate phosphoramidites $(R)-\mathbf{2}$ and $(R)-\mathbf{3}$ derived from $(R)$ BINOL is illustrated in Scheme 1. Treatment of the corresponding secondary amine with $\mathrm{PCl}_{3} / \mathrm{NEt}_{3}$ and subsequent reaction with $(R)-\mathbf{1}^{10}$ in the presence of $\mathrm{NEt}_{3}$ could provide the desired product as a white solid. The structures of $(R)-\mathbf{2}$ and $(R)-\mathbf{3}$ were confirmed by ${ }^{1} \mathrm{H}$ NMR, ${ }^{31} \mathrm{P}$ NMR spectra.

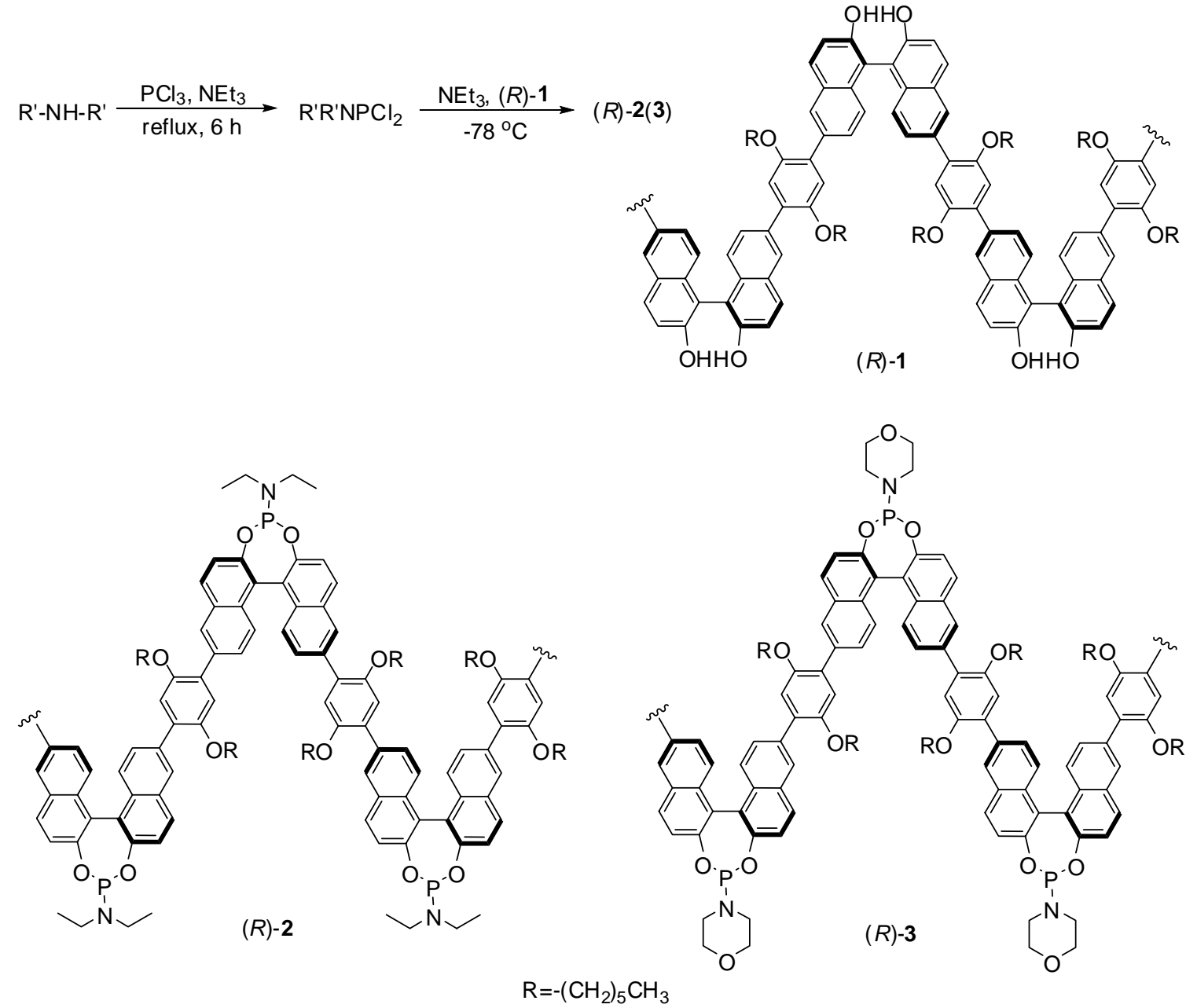

Scheme 1. Preparation of polymeric monodentate phosphoramidites ligands $(R)-\mathbf{2}$ and $(R)-\mathbf{3}$. 


\section{Asymmetric hydrogenation of $\alpha$-dehydroamino acid derivatives}

The conditions of asymmetric hydrogenation reaction catalyzed by $\mathrm{Rh} /(R)-2$ complex were optimized, and the results are listed in Table 1 . We found that the reaction carried out in THF gives higher yield and ee than that in dichloromethane. When the reaction was cooled to $5^{\circ} \mathrm{C}$, higher ee value was obtained (entries 3 and 5). Interestingly, the mole ratio of Rh/ligand played an important role in the catalytic hydrogenation. Unlike other reported asymmetric hydrogenation catalyzed by $\mathrm{Rh}$ - monodentate phosphine ligands, only moderate conversion and ee was obtained for the reaction with the mole ratio $1: 2$ of $\mathrm{Rh} /(R)-2$ (entry 2 ). Meanwhile, an insoluable yellow solid was deposited. The mole ratio $1: 1.1$ of $\mathrm{Rh} /(R)-\mathbf{2}$ afforded best catalytic results (entry 3). In order to investigate the effect of the $\mathrm{Rh} / \mathrm{L}$ ratio, we performed stoichiometric reactions with $\mathrm{Rh} / \mathrm{L}$ ratio from $2: 1$ to $1: 4$ at $5^{\circ} \mathrm{C}$ (entry 6-10). In agreement with earlier reports, ${ }^{5 f, 9 b, 14}$ The poorer catalytic activity was displayed when the reaction was catalyzed by a 1:3 or 1:4 Rh/L ratio. The yellow precipitate was observed in both cases. The reaction with $2: 1$ $\mathrm{Rh} / \mathrm{L}$ mole ratio gave exactly the same ee value than that of $1: 1 \mathrm{Rh} / \mathrm{L}$ mole ratio, but a black precipitate (rhodium black) was observed in the former reaction. In addition, the use of $\mathrm{Rh}(\mathrm{COD})_{2} \mathrm{SbF}_{6}$ instead of $\mathrm{Rh}(\mathrm{COD})_{2} \mathrm{BF}_{4}$ (entries 5 and 9) or higher $\mathrm{H}_{2}$ pressure (entries 5 and 11) seemed to have little effects on the reaction.

Table 1. Asymmetric Hydrogenation of Methyl (Z)-2-Acetamidocinnamate ${ }^{\mathrm{a}}$

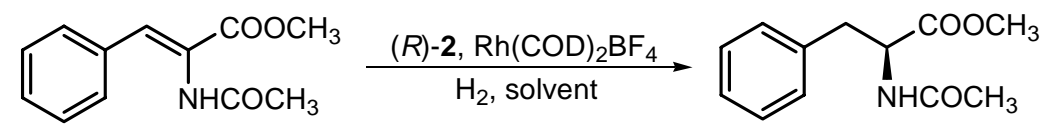

\begin{tabular}{cccccccc}
\hline Entry & Solvents & {$[\mathrm{Rh}]: \mathrm{L}$} & $\begin{array}{c}\mathrm{H}_{2} \\
(\mathrm{~atm})\end{array}$ & $\mathrm{T}\left({ }^{\circ} \mathrm{C}\right)$ & $\begin{array}{c}\mathrm{t} \\
(\mathrm{h})\end{array}$ & ${\text { Yield }(\%)^{\mathrm{b}}}$ & ee $(\%)^{\mathrm{c}}$ \\
\hline 1 & $\mathrm{CH}_{2} \mathrm{Cl}_{2}$ & $1: 2$ & 1.2 & 30 & 8 & 10 & nd \\
2 & $\mathrm{THF}$ & $1: 2$ & 1.2 & 30 & 8 & 86 & $84.3(S)$ \\
3 & $\mathrm{THF}$ & $1: 1.1$ & 1.2 & 30 & 8 & 100 & $92.5(S)$ \\
4 & $\mathrm{THF}$ & $1: 0.9$ & 1.2 & 30 & 8 & 100 & $92.1(S)$ \\
5 & THF & $1: 1.1$ & 1.2 & 5 & 8 & 100 & $95.6(S)$ \\
$6^{\mathrm{d}}$ & THF & $1: 4$ & 1.2 & 5 & 8 & 0 & nd \\
$7^{\mathrm{d}}$ & THF & $1: 3$ & 1.2 & 5 & 8 & 34.7 & $86.2(S)$ \\
$8^{\mathrm{d}}$ & THF & $1: 2$ & 1.2 & 5 & 8 & 82.5 & $86.4(S)$ \\
$9^{\mathrm{d}}$ & THF & $1: 1.1$ & 1.2 & 5 & 8 & 100 & $96.2(S)$ \\
$10^{\mathrm{d}}$ & THF & $2: 1$ & 1.2 & 5 & 8 & 100 & $96.8(S)$ \\
11 & THF & $1: 1.1$ & 4.0 & 5 & 8 & 100 & $95.2(S)$ \\
\hline
\end{tabular}

${ }^{\text {a }}$ Reactions were performed with $0.1 \mathrm{M}$ solutions of substrates with $\mathrm{S} / \mathrm{C}=100: 1$.

${ }^{b}$ Yields were determined by GC-MS. ${ }^{c}$ EE's were determined by HPLC on a Chiralcel OD-H column $^{15}$. The configurations of all the predominant products were in $S$ - form.

${ }^{\mathrm{d}} \mathrm{Rh}(\mathrm{COD})_{2} \mathrm{SbF}_{6}$ was used instead of $\mathrm{Rh}(\mathrm{COD})_{2} \mathrm{BF}_{4}$. 
The mechanism of rhodium-catalyzed asymmetric hydrogenation of functionalized olefins using monodentate phosphoramidite ligands has not been well addressed. The prevailing viewpoint was that two ligand molecules would bond to rhodium in the active catalytic species ${ }^{16}$. However, $\mathrm{Zhou}^{5 \mathrm{f}}$ and $\mathrm{Fan}^{9 \mathrm{~b}}$ found that in the asymmetric hydrogenation of (Z)-2(acetamido)cinnamate catalyzed by $\mathrm{Rh}$-monodentate phosphine, the reaction with 1:1 $\mathrm{Rh} / \mathrm{L}$ ratio was faster than that with $1: 2 \mathrm{Rh} / \mathrm{L}$ ratio, and the $\mathrm{Rh} / \mathrm{L}$ ratio had little influence on the conversion and enantioselectivity. Van den Berg ${ }^{14}$ proved that a number of different complexes are formed during hydrogenation.

Due to its unique structure, the polymeric ligands $(R)-2$ and $(R)-3$ with their rigid polymeric chains can prevent the coordination between $\mathrm{Rh}$ and two phosphoramidite units in one macromolecule. Thus, when reaction was processed with the $\mathrm{Rh} / \mathrm{L}$ ratio of $1: 2$, two phosphoramidite units from different polymer chains might bind to one $\mathrm{Rh}$ to form $\mathrm{RhL}_{2}$ species (Figure 1). It would result in a cross-linking reaction among the rigid polymer chains, and the catalyst would therefore deposit and lose its activity. When $\mathrm{Rh} /$ ligand ratio is $1: 1$, enough $\mathrm{Rh}$ atoms may prevent the cross-linking reaction among the linear rigid polymer chains, so the catalyst exhibits excellent reactivity and enantioselectivity in the catalytic applications.

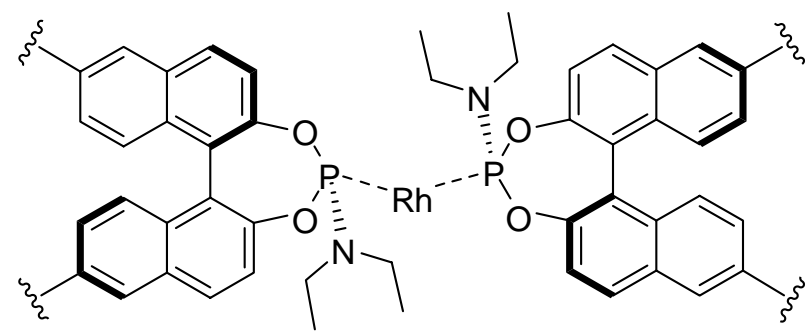

Polymeric chain A

Polymeric chain B

Figure 1. Fragment of the product of supposed cross-linking reaction among the polymeric chains of $(R)-2$ with $1: 2 \mathrm{Rh} / \mathrm{L}$ ratio.

The asymmetric hydrogenation of other $\alpha$-dehydroamino acid derivatives catalyzed by $\mathrm{Rh} /(R)-\mathbf{2}$ was also investigated under the optimized conditions. The results are listed in Table 2. All of the tested substrates could be completely converted to the desired products with excellent enantioselectivities $(92-99 \%$ ee), which were comparable to those obtaining with the corresponding monomeric ligand $\left(97 \%\right.$ ee) ${ }^{5 \mathrm{j}}$ The morpholine-containing ligand $(R)-3$ exhibited relatively lower enantioselectivity (88-91\% ee) in comparison with its homogeneous counterpart $\left(98 \%\right.$ ee) ${ }^{5 \mathrm{j}}$ After the completion of the reaction, these polymeric Rh complexes could be easily precipitated through the addition of methanol. Unfortunately, due to the change in the structure of the Rh complex, the solid that separated from the reaction system could not be reused for another catalytic round. 
Table 2. Asymmetric hydrogenation of $\alpha$-dehydroamino acid derivatives ${ }^{\mathrm{a}}$

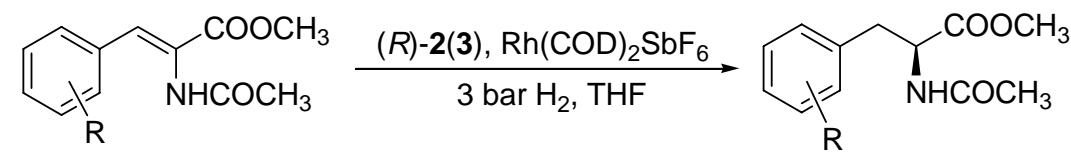

\begin{tabular}{llllll}
\hline Entry & $\mathrm{L}$ & $\mathrm{R}$ & $\mathrm{t}(\mathrm{h})$ & ${\text { Yield }(\%)^{\mathrm{b}}}$ & $\mathrm{Ee}(\%)^{\mathrm{c}}$ \\
\hline 1 & $(R)-2$ & $\mathrm{H}$ & 8 & 100 & 96.2 \\
2 & $(R)-2$ & $2-\mathrm{CH}_{3}$ & 8 & 100 & 99.5 \\
3 & $(R)-2$ & $4-\mathrm{CH}_{3}$ & 8 & 100 & 97.8 \\
4 & $(R)-2$ & $4-\mathrm{OCH}_{3}$ & 8 & 100 & 92.1 \\
5 & $(R)-2$ & $4-\mathrm{F}$ & 8 & 100 & 99.0 \\
6 & $(R)-2$ & $4-\mathrm{Cl}$ & 8 & 100 & 99.2 \\
7 & $(R)-2$ & $4-\mathrm{Br}$ & 8 & 100 & 97.1 \\
8 & $(R)-3$ & $\mathrm{H}$ & 8 & 100 & 88.5 \\
9 & $(R)-3$ & $2-\mathrm{CH}_{3}$ & 8 & 100 & 91.3 \\
10 & $(R)-3$ & $4-\mathrm{CH}_{3}$ & 8 & 100 & 90.5 \\
11 & $(R)-3$ & $4-\mathrm{OCH} H_{3}$ & 8 & 100 & 86.6 \\
12 & $(R)-3$ & $4-\mathrm{F}$ & 8 & 100 & 90.1 \\
13 & $(R)-3$ & $4-\mathrm{Cl}$ & 8 & 100 & 90.3 \\
14 & $(R)-3$ & $4-\mathrm{Br}$ & 8 & 100 & 89.9 \\
\hline
\end{tabular}

${ }^{a}$ The reaction was performed with $0.1 \mathrm{M}$ solutions of substrates with $\mathrm{S} / \mathrm{C}=100: 1$ at $5^{\circ} \mathrm{C}$ in 5 $\mathrm{mL}$ of THF, $[\mathrm{Rh}] / \mathrm{L}=1: 1.1{ }^{\mathrm{b}}$ Yields were determined by GC-MS.

' Ee's and conversions were determined by HPLC on a Chiralcel OD-H column ${ }^{15}$. The configurations of all the predominant products were in $S$ - form.

\section{Conclusions}

In summary, we developed a new class of polymeric monodentate phosphoramidite ligands with rigid and sterically regular polybinaphthyl structure as a backbone. Their rhodium complexes were successfully applied in the asymmetric hydrogenation of $\alpha$-dehydroamino acid derivatives. Comparable enantioselectivities were achieved as compared to that obtained from MonoPhos. The best $\mathrm{Rh} / \mathrm{L}$ ratio for asymmetric hydrogenation was 1:1 instead of 1:2. Increase of the amount of ligand led to the formation of precipitate and the conversion as well as the enantioselectivity decrease. 


\section{Experimental Section}

General Procedures. The ${ }^{1} \mathrm{H}$ NMR spectra were measured on a Varian INOVA-400 spectrometer and the chemical shifts were referenced to tetramethylsilane $\left({ }^{1} \mathrm{H}\right)$ as internal standard or $\mathrm{H}_{3} \mathrm{PO}_{4}\left({ }^{31} \mathrm{P}\right)$ as external standard. GC-MS spectra data were recorded on a Agilent $6890-5973 \mathrm{~N}$ spectrometer with a HP-5 $(30 \mathrm{~m} \times 0.25 \mathrm{~mm})$ column. All reactions were performed under argon atmosphere, using standard Schlenk techniques. Solvents were dried and distilled before use following standard procedures. Chemicals and reagents were purchased from commercial suppliers and used as received.

\section{Copolymer (R)-1}

This material was prepared following literature procedures. ${ }^{10}$ Pale yellow solid in $80 \%$ yield. GPC analysis showed the molecular weight as $\mathrm{M}_{\mathrm{w}}=16,981$ and $\mathrm{M}_{\mathrm{n}}=11,305(\mathrm{PDI}=1.50)$. [ $\left.\alpha\right]_{\mathrm{D}}$ $=-397.2^{\circ}\left(\mathrm{c}=1.00, \mathrm{CHCl}_{3}\right) .{ }^{1} \mathrm{H} \mathrm{NMR}\left(\mathrm{CDCl}_{3}, 400 \mathrm{MHz}\right): \delta=8.16(\mathrm{~m}, 2 \mathrm{H}), 8.01(\mathrm{~d}, \mathrm{~J}=8.3 \mathrm{~Hz}$, 2H), $7.60(\mathrm{~d}, \mathrm{~J}=8.6 \mathrm{~Hz}, 2 \mathrm{H}), 7.40(\mathrm{~d}, \mathrm{~J}=8.3 \mathrm{~Hz}, 2 \mathrm{H}), 7.26(\mathrm{~d}, \mathrm{~J}=8.6 \mathrm{~Hz}, 2 \mathrm{H}), 7.11(\mathrm{~s}, 2 \mathrm{H})$, $3.94(\mathrm{~m}, 6 \mathrm{H}), 1.62-1.72(\mathrm{~m}, 4 \mathrm{H}), 1.35(\mathrm{~m}, 4 \mathrm{H}), 1.24-1.36(\mathrm{~m}, 8 \mathrm{H}), 0.80(\mathrm{~s}, 6 \mathrm{H})$.

\section{Polymeric monodentate phosphoramidite (R)-2}

Under argon atmosphere, a solution of diethylamine $(0.05 \mathrm{~mL}, 0.48 \mathrm{mmol})$ and triethylamine $(0.074 \mathrm{~mL}, 0.52 \mathrm{mmol})$ in THF $(2 \mathrm{~mL})$ was added dropwise to the solution of phosphorus trichloride $(0.042 \mathrm{~mL}, 0.48 \mathrm{mmol})$ in THF $(2 \mathrm{~mL})$. The mixture was stirred at $70^{\circ} \mathrm{C}$ for $6 \mathrm{~h}$ and allowed to cool to room temperature. Triethylamine $(0.074 \mathrm{~mL}, 0.52 \mathrm{mmol})$ was then added dropwise, and the solution was cooled to $-78^{\circ} \mathrm{C}$. A solution of $(R)-1(0.27 \mathrm{~g}, 0.48 \mathrm{mmol}$ based on the repeated unit) in THF $(4 \mathrm{~mL})$ was added dropwise. The resulted mixture was allowed to warm up slowly to room temperature and was stirred for additional $12 \mathrm{~h}$. The mixture was filtered and the filtrate was concentrated to $3 \mathrm{~mL}$ under reduced pressure. $\mathrm{MeOH}(3 \mathrm{~mL})$ was added to the residue to precipitate the product polymer as a white solid. Yield: $0.21 \mathrm{~g}(65 \%)$. $[\alpha]_{\mathrm{D}}$ $=-434.9^{\circ}\left(\mathrm{c}=1.00, \mathrm{CHCl}_{3}\right) .{ }^{1} \mathrm{H}-\mathrm{NMR}\left(\mathrm{CDCl}_{3}, 400 \mathrm{MHz}\right): \delta=8.17(\mathrm{~s}, 2 \mathrm{H}), 7.92-8.01(\mathrm{~m}, 2 \mathrm{H})$, 7.40-7.60 (m, 4H), $7.27(\mathrm{~m}, 2 \mathrm{H}), 7.10(\mathrm{~m}, 2 \mathrm{H}), 3.96(\mathrm{~m}, 4 \mathrm{H}), 3.0-3.08(\mathrm{~m}, 4 \mathrm{H}), 1.35-1.68(\mathrm{~m}$, $8 \mathrm{H}), 1.18(\mathrm{~m}, 8 \mathrm{H}), 1.07-1.09(\mathrm{~m}, 6 \mathrm{H}), 0.74(\mathrm{~s}, 6 \mathrm{H}) .{ }^{31} \mathrm{P}-\mathrm{NMR}: \delta=149.79$. Anal. Calcd. for $\mathrm{C}_{42} \mathrm{H}_{48} \mathrm{NO}_{4} \mathrm{P}: \mathrm{C}, 76.22 ; \mathrm{H}, 7.31 ; \mathrm{N}, 2.12$; found: C, 75.46; H, 6.60; N, 2.42.

\section{Polymeric monodentate phosphoramidite (R)-3}

The title compound was prepared from morpholine following the preparation of $(R)-\mathbf{2}$ as a pale yellow solid. Yield: $66 \%$. $[\alpha]_{\mathrm{D}}=-337.2^{\circ}\left(\mathrm{c}=1.00, \mathrm{CHCl}_{3}\right)$. H-NMR $\left(\mathrm{CDCl}_{3}, 400 \mathrm{MHz}\right): \delta=$ 8.22-8.00 (m, 4H), 7.66-7.28 (m, 6H), 7.13-7.18 (m, 2H), 3.97 (4H), 3.40-3.65 (m, 4H), 3.04$3.16(\mathrm{~m}, 4 \mathrm{H}), 1.71(\mathrm{~m}, 4 \mathrm{H}), 1.38(\mathrm{~m}, 4 \mathrm{H}), 1.24-1.30(\mathrm{~m}, 8 \mathrm{H}), 0.79(\mathrm{~s}, 6 \mathrm{H}) .{ }^{31} \mathrm{P}-\mathrm{NMR}: \delta=144.86$. Anal.Calcd. for $\mathrm{C}_{42} \mathrm{H}_{46} \mathrm{NO}_{5} \mathrm{P}: \mathrm{C}, 74.65 ; \mathrm{H}, 6.86 ; \mathrm{N}, 2.07$; found: $\mathrm{C}, 74.17 ; \mathrm{H}, 7.24 ; \mathrm{N}, 1.70$. 


\section{General procedure for the $\mathbf{R h}(\mathrm{I})$-catalyzed asymmetric hydrogenation}

The catalyst was formed in situ by mixing $\left[\mathrm{Rh}(\mathrm{COD})_{2}\right] \mathrm{BF}_{4}$ or $\left[\mathrm{Rh}(\mathrm{COD})_{2}\right] \mathrm{SbF}_{6}(0.005 \mathrm{mmol})$ and the polymeric monodentate phosphoramidite ligands $(0.0055 \mathrm{mmol})$ in THF $(5 \mathrm{~mL})$ for 10 min. The clear catalyst solution was transferred into a $100 \mathrm{~mL}$ stainless steel autoclave, which contained $\alpha$-dehydroamino acid derivatives $(0.5 \mathrm{mmol})$ and a magnetic stirring bar, through a glass liner. After purging with hydrogen for 6 times, the system was pressurized with hydrogen ( 5 bar) and the reaction mixture was stirred for $8 \mathrm{~h}$. Then the reaction mixture was filtered over a short pad of gel and analyzed by HPLC. ${ }^{15}$ All of the catalytic experiments were independently repeated 2 times to confirm the results.

\section{Enantiomeric excess determinations}

The racemic mixtures of all products were prepared by hydrogenation of the substrates using $\mathrm{Pd} / \mathrm{C}$ or Wilkinson's catalyst.

(S)-Methyl 2-acetamido-3-phenylpropionate. 95.8\% ee, (HPLC, Daicel Chiralcel OD-H, 0.5 $\mathrm{mL} / \mathrm{min}, 10 \%$ isopropanol $/$ hexane, $(\mathrm{R}) \mathrm{t}_{1}=22.485 \mathrm{~min} ;(\mathrm{S}) \mathrm{t}_{2}=28.882 \mathrm{~min}$ ).

(S)-Methyl 2-acetamido-3-(2-methylphenyl)propionate. 99\% ee, (HPLC, Daicel Chiralcel OD-H, $0.5 \mathrm{~mL} / \mathrm{min}, 10 \%$ isopropanol/hexane, $(\mathrm{R}) \mathrm{t}_{1}=21.4 \mathrm{~min}$; $(\mathrm{S}) \mathrm{t}_{2}=27.5 \mathrm{~min}$ ).

(S)-Methyl 2-acetamido-3-(4-methylphenyl)propionate. 98\% ee, (HPLC, Daicel Chiralcel OD-H, $0.5 \mathrm{~mL} / \mathrm{min}, 10 \%$ isopropanol/hexane, $(\mathrm{R}) \mathrm{t}_{1}=19.6 \mathrm{~min}$; $\left.(\mathrm{S}) \mathrm{t}_{2}=25.4 \mathrm{~min}\right)$.

(S)-Methyl 2-acetamido-3-(4-methoxyphenyl)propionate. 92\% ee, (HPLC, Daicel Chiralcel OD-H, $0.5 \mathrm{~mL} / \mathrm{min}, 10 \%$ isopropanol/hexane, $(\mathrm{R}) \mathrm{t}_{1}=29.0 \mathrm{~min}$; $(\mathrm{S}) \mathrm{t}_{2}=36.1 \mathrm{~min}$ ).

(S)-Methyl 2-acetamido-3-(4-Fluorophenyl)propionate. 99\% ee, (HPLC, Daicel Chiralcel OD-H, $0.5 \mathrm{~mL} / \mathrm{min}, 10 \%$ isopropanol/hexane, $\left.(\mathrm{R}) \mathrm{t}_{1}=23.3 \mathrm{~min} ;(\mathrm{S}) \mathrm{t}_{2}=29.0 \mathrm{~min}\right)$.

(S)-Methyl 2-acetamido-3-(4-chlorophenyl)propionate. 99\% ee, (HPLC, Daicel Chiralcel OD$\mathrm{H}, 0.5 \mathrm{~mL} / \mathrm{min}, 10 \%$ isopropanol $/$ hexane, $\left.(\mathrm{R}) \mathrm{t}_{1}=26.4 \mathrm{~min} ;(\mathrm{S}) \mathrm{t}_{2}=32.1 \mathrm{~min}\right)$.

(S)-Methyl 2-acetamido-3-(4-bromophenyl)propionate. 97.2\% ee, (HPLC, Daicel Chiralcel OD-H, $0.5 \mathrm{~mL} / \mathrm{min}, 10 \%$ isopropanol/hexane, $(\mathrm{R}) \mathrm{t}_{1}=28.8 \mathrm{~min}$; $\left.(\mathrm{S}) \mathrm{t}_{2}=35.6 \mathrm{~min}\right)$.

\section{Acknowledgements}

This work was financially supported by the National Science Foundation of China (Nos. 20725206, 20732004 and 20572075), Program for New Century Excellent Talents in University, Specialized Research Fund for the Doctoral Program of Higher Education and Scientific Fund of Sichuan Province for Outstanding Young Scientist.

\section{References}

1. (a) Knowles, W. S. Angew. Chem., Int. Ed. 2002, 41, 1998. (b) Noyori, R. Angew. Chem., 
Int. Ed. 2002, 41, 2008. (c) Chaloner, P. A.; Esteruelas, M. A.; Joo, F.; Oro, L. A. Homogeneous Hydrogenation; Kluwer: Dordrecht, 1994. (d) Jacobsen, E. N.; Pfaltz, A.; Yamamoto, H., Eds. In Comprehensive Asymmetric Catalysis; Springer: Berlin, 1999; Vol. I-III. (e) Tungler, A.; Sípos, E.; Háda, V. Arkivoc 2004, (vii), 223.

2. Noyori, R. Asymmetric Catalysis in Organic Synthesis; Wiley \& Sons: New York, 1994; Chapter 2.

3. Tang, W.; Zhang, X. Chem. Rev. 2003, 103, 3029.

4. (a) Reetz, M. T.; Sell, T. Tetrahedron Lett. 2000, 41, 6333. (b) van den Berg, M.; Minnaard, A. J.; Schudde, E. P.; van Esch, J.; de Vries, A. H. M.; de Vries, J. G.; Feringa, B. L. J. Am. Chem. Soc. 2000, 122, 11539. (c) Reetz, M. T.; Mehler, G. Angew. Chem. Int. Ed. 2000, 39, 3889. (d) Peña, D. M.; Minnaard, A. J.; de Vries, J. G.; Feringa, B. L. J. Am. Chem. Soc. 2002, 124, 14552.

5. See for example: (a) Chen, W.; Xiao, J. Tetrahedron Lett. 2001, 42, 8737. (b) Hannen, P.; Militzer, H.-C.; Vogl, E. M.; Rampf, F. A. Chem. Commun. 2003, 2210. (c) Huang, H.; Zheng, Z.; Luo, H.; Bai, C.; Hu, X.; Chen, H. J. Org. Chem. 2004, 69, 2355. (d) Jia, X.; Li, X.; Xu, L.; Shi, Q.; Yao, X.; Chan, A. S. C. J. Org. Chem. 2003, 68, 4539. (e) Hua, Z.; Vassar, V. C.; Ojima, I. Org. Lett. 2003, 5, 3831. (f) Fu, Y.; Guo, X.-X.; Zhu, S.-F.; Hu, A.-G.; Xie, J.-H.; Zhou, Q.-L. J. Org. Chem. 2004, 69, 4648. (g) Jerphagnon, T.; Renaud, J.-L.; Demonchaux, P.; Ferreira, A.; Bruneau, C. Adv. Synth. Catal. 2004, 346, 33. (h) Liu, Y.; Ding, K. J. Am. Chem. Soc. 2005, 127, 10488. (i) Reetz, M. T.; Li, X. Synthesis 2005, 3183. (j) Bernsmann, H.; van den Berg, M.; Hoen, R.; Minnaard, A. J.; Mehler, G.; Reetz, M. T.; de Vries, J. G.; Feringa, B. L. J. Org. Chem. 2005, 70, 943. (k) Liu, Y.; Sandoval, C. A.; Yamaguchi, Y.; Zhang, X.; Wang, Z.; Kato, K.; Ding, K.-L. J. Am. Chem. Soc. 2006, 128, 14212; (1) Eberhardt, L.; Armspach, D.; Matt, D.; Toupet, L.; Oswald, B. Eur. J. Inorg. Chem. 2007, 4153. (m) Monti, C.; Gennari, C.; Piarulli, U. Tetrahedron Lett. 2004, 45, 6859. (n) Reetz, M. T.; Ma, J.-A.; Goddard, R. Angew. Chem. Int. Ed. 2005, 44, 412. (o) Boogers, J. A. F.; Felfer, U.; Kotthaus, M.; Lefort, L.; Steinbauer, G.; de Vries, H. M.; de Vries, J. G. Org. Proc. Res. Dev. 2007, 11, 585. (p) Minnaard, A. J.; Feringa, B.; Lefort, L.; de Vries, J. G. Acc. Chem. Res. 2007, 40, 1267. (q) Zalubovskis, R.; Hörmann, E.; Pfaltz, A.; Moberg, C. Arkivoc 2008, (xiv), 58. (r) Yang, M.; Yang, X.-B.; Yu,X.-Q. Arkivoc 2008, (xvi), 84.

6. (a) Chen, W.-P.; Roberts, S. M.; Whittall, J. Tetrahedron Lett. 2006, 47, 4263. (b) Doherty, S.; Robins, E. G.; Pál, I.; Newman, C. R.; Hardacre, C.; Rooney, D.; Mooney, D. A. Tetrahedron: Asymmetry 2003, 14, 1517. (c) Huttenloch, O.; Laxman, E.; Waldmann, H. Chem. Commun. 2002, 673. (d) Huttenloch, O.; Laxman, E.; Waldmann, H. Chem. Eur. J. 2002, 8, 4767. (e) Wang, X.; Ding, K. J. Am. Chem. Soc. 2004, 126, 10524. (f) Hu, X.; Huang, J.; Zeng, Q.; Zheng, Z. Chem. Commun. 2006, 293. (g) Hoen, R.; Leleu, S.; Botman, P. N. M.; Appelman, V. A. M.; Feringa, B. L.; Hiemstra, H.; Minnaard, A. J.; van Maarseveen, J. H. Org. Biomol. Chem. 2006, 4, 613.

7. Sherrington, D. C. Chem. Commun. 1998, 2275. 
8. Tomalia, D. A.; Dvornic, P. R. Nature 1994, 372, 617.

9. (a) Botman, P. N. M.; Amore, A.; van Heerbeek, R.; Back, J. W.; Hiemstra, H.; Reek, J. N. H.; van Maarseveen, J. H. Tetrahedron Lett. 2004, 45, 5999. (b) Tang, W.-J.; Huang, Y.-Y.; He, Y.-M.; Fan, Q.-H. Tetrahedron Asymmetry 2006, 17, 536.

10. Huang, W.-S.; Hu, Q.-S.; Zheng, X.-F.; Anderson, J.; Pu, L. J. Am. Chem. Soc. 1997, 119, 4313.

11. Huang, W.-S.; Hu, Q.-S.; Zheng, X.-F.; Pu, L. J. Org. Chem. 1998, 63, 1364.

12. Yu, H.-B.; Hu, Q-S.; Pu, L. Tetrahedron Lett. 2000, 41,1681.

13. Yu, H.-B.; Hu, Q-S.; Pu, L. J. Am. Chem. Soc. 2000, 122, 6500.

14. van den Berg, M.; Minnaard, A. J.; Haak, R. M.; Leeman, M.; Schudde, E. P.; Meetsma, A.; Feringa, B. L.; de Vries, A. H. M.; Maljaars, C. E. P.; Willans, C. E.; Hyett, D.; Boogers, J. A. F.; Henderickx, H. J. W.; de Vries, J. G. Adv. Synth. Catal. 2003, 345, 308.

15. (a) Navarre, L.; Martinez, R.; Genet, J. P.; Darses, S. J. Am. Chem. Soc. 2008, 130, 6159. (b) Wei, H.; Zhang, Y.-J.; Dai, Y.-J.; Zhang, J.-M.; Zhang, W.-B. Tetrahedron Lett. 2008, 494106.

16. Reetz, M. T.; Meiswinkel, A.; Mehler, G.; Angermund, K.; Graf, M.; Thiel, W.; Mynott, R.; Blackmond, D. G. J. Am. Chem. Soc. 2005, 127, 10305. 